morphology, seed mass and shade tolerance in seedlings of nine boreal tree species grown in high and low light. Funct Ecol 12: 327-338.

Reich, P. B., I. J. Wright and J. Cavender-Bares et al. (2003): The evolution of plant functional variation: traits, spectra, and strategies. Int J Plant Sci 164: S143-S164.

Simpson, W. T. (1993): Specific gravity, moisture content, and density relationship for wood. Gen. Tech. Rep. FPL-GTR 76, Madison WI: U.S. Department of Agriculture, Forest Service, Forest Products Laboratory. $13 \mathrm{p}$.

SMith, D. M. (1954): Maximum moisture content method for determining specific gravity of small wood samples. Report No. 2014, Madison WI: U.S. Department of Agriculture, Forest Service, Forest Products Laboratory.

Steele, M. J., M. P. Coutts and M. M. Yeoman (1989): Developmental changes in Sitka spruce as indices of physiological age I. Changes in needle morphology. New Phytol. 113: 367-375.

Stellrecht, J. W., C. A. Mohn and W. Cromell (1974): Productivity of white spruce seed sources in a Minnesota tree planting. Minnesota For Res Notes No. 251.
Weng, Y., K. Tosh and M. Fullarton (2010): Determining and projecting realised genetic gains: results from early-stage spruce improvement programmes in New Brunswick, Canada. New Zeal J For Sci 40: 5-17.

White, T. L. and G. R. Hodge (1989): Predicting breeding values with applications in tree improvement. Kluwer Academic Publishers, Dordrecht. The Netherlands.

Wilkinson, R. C. (1977): Inheritance of budbreak and correlation with early height growth in white spruce (Picea glauca) from New England. NE Exp Stn Res Pap-391, U.S. Department of Agriculture, Forest Service, Upper Darby, PA.

ZHANG, S. Y. (1995): Effect of growth rate on wood specific gravity and selected mechanical properties in individual species from distinct wood categories. Wood Sci Technol 29: 451-465.

Zhang, S. Y., Q. Yu and J. Beaulieu (2004): Genetic variation in veneer quality and its correlation to growth in white spruce. Can J For Res 34: 1311-1318.

\title{
Use of genetic markers to build a new generation of Eucalyptus pilularis breeding population
}

\author{
By P. H. M. DA Silva ${ }^{1), *)}$, M. ShePherd ${ }^{2)}$, D. Grattapaglia ${ }^{3)}$ and A. M. SebbenN ${ }^{4)}$
}

(Received 21 $1^{\text {th }}$ July 2015)

\begin{abstract}
Tree improvement generally proceeds by incremental gains obtained from recurrent selection in large diverse populations but is

\footnotetext{
1) Instituto de Pesquisas e Estudos Florestais (IPEF), Avenida Pádua Dias 11, Caixa Postal 530, CEP 13400970, Piracicaba, SP, Brazil.

2) Southern Cross Plant Science, Southern Cross University, PO Box 157, Lismore, NSW 2480, Australia.

$\left.{ }^{3}\right)$ Plant Genetics Laboratory, EMBRAPA Genetic Resources and Biotechnology, CEP 70770-970, DF, Brasilia, Brazil, and Graduate Program in Genomic Sciences Biotechnology and Universidade Católica de Brasília, SGAN Qd 916, CEP 70790-160, DF, Brasília, Brazil.
}

slow due to long generation times and delay till trees reach assessment age. This places a premium upon extracting data from historic introductions used to found landraces when reinstating modern breeding programs. The value of such resources, however, may be

\footnotetext{
4) Instituto Florestal de São Paulo, CP 1322, São Paulo, SP, 01059-970, Brazil; and Faculdade de Engenharia de Ilha Solteira/UNESP, Ilha Solteira, SP, Brazil, Caixa Postal, 31, 15385-000.

*) Corresponding author: PAUlo H.M. DA Silva. Instituto de Pesquisas e Estudos Florestais (IPEF), Avenida Pádua Dias 11, Caixa Postal 530, CEP 13400-970, Piracicaba, SP, Brazil. Phone: 5519 210-58600. Mail: paulohenrique@ipef.br
} 
degraded due to a lack of records on germplasm origins, pedigrees and early performance, but DNA technology may help recoup some of this value. Eucalyptus pilularis (subgenus Eucalyptus) is regarded as a premier hardwood plantation species for saw log and poles in Australia, but has not been used extensively despite early introductions and testing in many countries overseas. Here we use DNA fingerprinting to assess genetic diversity and inbreeding in historic introductions of $E$. pilularis to evaluate this resource in advance of a reinvigorated breeding effort for this species in Brazil. As expected, based on the available documentation for the introductions, genetic diversity relative to Australian reference populations does not appear to be compromised, and there was unlikely to be excessive inbreeding. Also, favorable, was the likelihood that further selections should not unduly increase the relationship in the next generation. Interestingly, we note the importance of testing widely adapted sources of germplasm when making introductions, as provenances which performed poorly in tests on productive sites in Australia, may have value when matched with lower fertility sites overseas.

Key words: Blackbutt, genetic diversity, relatedness, growth, pedigree information.

\section{Introduction}

The majority of eucalypt plantations worldwide are based on nine species that belongs to subgenera Symphyomyrtus: E. camaldulensis, $E$. dunnii, E. globulus, E. grandis, E. nitens, $E$. pellita, E. saligna, E. tereticornis e E. urophylla (HARWOOD, 2011), due to high productivity, vegetative propagation, plasticity and wood traits quality for specific utility (GONÇALVES et al., 2013). However there are others species of eucalypts with high potential for commercial plantation that belongs to different subgenera, like $E$. pilularis. Classics studies indicate that the species has good growth and has wide potential for sawing (PASZTOR, 1974; ALCORN et al., 2008).

Eucalyptus pilularis (blackbutt), belongs to subgenus Eucalyptus (subgenus Eucalyptus) and Renantheria section and occurs in New South Wales in the coastal plains, the mountainous areas near the coast, extending to south of Queensland (BROOKER and KLEING, 2006). In Brazil the species was investigated in the end of 70 's and begins of 80 's, when a few breeding populations by different institutions were set up, however the reasons for non-use intensively are: i) inability to hybridize with widely used species (E. urophylla and E. grandis); ii) inability to resprout (no coppice management); and iii) the wood quality, which is not interesting for some purposes (e.g. pulp and charcoal production).

The most important aspect for selection of commercial genotypes is the productivity relative to environmental conditions (STAPE et al., 2008), due to the effects the environment has on growth, biomass production and harvestable yield (JALEEL et al., 2009). Another important aspect that affects productivity is the provenance. For $E$. pilularis, the highest productive provenances are related with good environmental conditions of the origin (BURGESS, 1975). Eucalyptus pilularis in natural area shows low regional genetic differentiation, high but uniform genetic diversity (SHEPHERD et al., 2010). In $E$. pilularis populations there is substantial genetic variation which will allow for the possibility to select for breeding. (CARNEGIE et al., 2004).

To compose a breeding population it is necessary to select plus trees with desired traits and high genetic variability. Normally, high intensity selection to find productive genotypes will decrease the genetic base. Therefore, it is important to know the genetic diversity of the breeding population, to avoid further genetic loss and buildup of inbreeding. Managing these aspects of breeding is challenging in situations where there is a need to use early introductions of trees which have been archived in germplasm resource collections without detailed records of the seed parent sources or tree identities. In some cases, the degree of relationship among trees is unknown, therefore use of genetic markers may be valuable in identifying the natural origins (provenance) of trees, the degree of diversity and the relationships among trees in a population (KUMAR and RichaRdSON, 2005; El-KASSABY et al., 2011).

The aim of the present study was to evaluate the potential of genetic markers (microsatellites) to aid in the selection of trees to form a new population for breeding of $E$. pilularis in Brazil. We genotyped a total 126 trees using 21 microsatellite loci from 9 populations established in Brazil during the 1970s and 80s, that are the base of a new breeding population. 


\section{Material and Methods}

\subsection{Materials, sampling and growth measurements}

The study uses a collection of nine E. pilularis populations planted by Brazilian forestry companies and the University of São Paulo in the States of São Paulo and Minas Gerais in Brazil during the 1970's and 1980, except for the Feena population which was planted in 1919. These trials were established across a range of years and at different locations and climatic conditions (Table 1). The number of provenances represented in each trial ranged from 1 to 13 and are documented in the Supplementary materials (Tables 1,2 and 3). For trials Anh-82, Anh-103, Ita1-67 and Ita-498 it was known that material was sourced from multiple provenances and that several trees per family were planted in the trials, however, the provenance identity of individual trees in the trials was not retained and is currently unavailable. The experimental design of trials was also lost over time and locations of trees was lost due to undocumented thinning, so the relationships amongst the trees is unknown. All populations studied are first generation introductions into Brazil, except for two seedlots in both Anhembi multiple provenances populations (from MogiGuaçu, BR and Zimbabwe) and for the Feena population, which is thought to be from seed introduced around 115 years ago, and a $2^{\text {nd }}$ generation population.

In 2013 seed capsules were collected from most of trees (with the exception of the Rio Claro population where no seed was available) to allow the development of a new base population for breeding of $E$. pilularis in Brazil. Capsules were air dried and seed extracted and stored under controlled conditions $\left(\mathrm{T}=10^{\circ} \mathrm{C}\right.$ and humidity $=50 \%$ ). Seed collections were made from individual trees (progenies) that were considered plus trees because of their phenotypic performance (growth and shape). In addition, leaves from each tree were sampled to allow genetic characterization and its diameter at breast height $(\mathrm{DBH})$ and height were also measured (Table 1).

In addition to the nine Brazilian collections, DNA from 22 trees sampled in natural stands

Table 1. - Description of Eucalyptus pilularis Brazilian and Australian provenances and climatic characteristics of Brazilian provenances.

\begin{tabular}{|c|c|c|c|c|c|c|c|c|}
\hline \multicolumn{2}{|c|}{ Population } & \multirow{2}{*}{$\frac{\text { Institution }}{\text { USP }}$} & \multirow{2}{*}{$\frac{\text { City }}{\text { Anhembi, SP }}$} & \multirow{2}{*}{$\begin{array}{c}\text { Planted } \\
1982\end{array}$} & \multirow{2}{*}{$\frac{\text { Code }}{7 \mathrm{~B} 082}$} & \multirow{2}{*}{\multicolumn{2}{|c|}{$\frac{\text { Prowenance }}{\text { Multiprovenace }^{* * * * *}}$}} & \multirow{2}{*}{$\frac{\text { Sample }}{16}$} \\
\hline 1 & Anh-82 & & & & & & & \\
\hline 2 & Anl1-103* & USP & Anhembi, $S P$ & 1985 & $7 C .103$ & \multicolumn{2}{|c|}{ Multiprovenace*** } & 6 \\
\hline 3 & Anh- 145 & USP & Anhembi, SP & 1989 & $7 D 145$ & \multicolumn{2}{|c|}{ Frascr Island } & 15 \\
\hline 4 & Feena* & FEENA & Rio Claro, SP & 1919 & $15 a$ & \multicolumn{2}{|c|}{ Comp. Paulista } & 5 \\
\hline 5 & Ita- 176 & Aperam & Itamarandiba, MG & 1984 & 176 & \multicolumn{2}{|c|}{ Beerburrum } & 15 \\
\hline 6 & $\operatorname{lta}-498$ & Apcram & Itamarandiba, MG & 1985 & 498 & \multicolumn{2}{|c|}{$\begin{array}{l}\text { Conglomerate, Woolgoolga, } \\
\text { Gallangowan, Whian Whian }\end{array}$} & 22 \\
\hline 7 & Ita-167 & Aperam & Itamarandiba, $\mathrm{MG}$ & 1984 & 167 & \multicolumn{2}{|c|}{ Multiprovenace**** } & 17 \\
\hline 8 & Bir-K & Suzano & Biritiba Mirim, SP & 1976 & $\mathrm{G} 5 \mathrm{~A}$ & \multicolumn{2}{|c|}{ Kiwarrak NSW } & 15 \\
\hline 9 & $\mathrm{Bit}-\mathrm{C}$ & Suzano & Biritiba Mirim, SP & 1976 & G5A & \multicolumn{2}{|c|}{ Coopemook, NSW } & 15 \\
\hline 10 & S-Aus & $\mathrm{SCU}$ & South Austrália & Natural & ND & \multicolumn{2}{|c|}{ Multi } & 16 \\
\hline 11 & $\mathrm{~N}-\mathrm{Aus}$ & $\mathrm{SCU}$ & North Austrália & Natural & NI) & \multicolumn{2}{|c|}{ Congloneral } & 6 \\
\hline \multicolumn{2}{|r|}{ City } & $\begin{array}{c}\text { Köppen } \\
\text { classification }\end{array}$ & \multicolumn{2}{|c|}{$\begin{array}{l}\text { Mean } \\
\text { temperature }\end{array}$} & $\begin{array}{l}\text { Rain fall } \\
\text { (mm) }\end{array}$ & $\begin{array}{l}\text { Altitude } \\
\text { (m) }\end{array}$ & Lat S & Long W \\
\hline \multicolumn{2}{|c|}{ Rio Claro, SP } & Cwa & \multicolumn{2}{|l|}{$21.6^{\circ} \mathrm{C}$} & 1366 & 620 & $22^{\circ} 14$ & $47^{\circ} 18$ \\
\hline \multicolumn{2}{|c|}{ Anhembi, $S P^{* *}$} & $\mathrm{Aw}$ & \multicolumn{2}{|l|}{$22.3^{\circ} \mathrm{C}$} & 1307 & 480 & $22^{\prime \prime} 28$ & $48^{(1)} 04$ \\
\hline & Mirim, SP & Cw & $19.8^{\circ} \mathrm{C}$ & & 1364 & 780 & $23^{10} 20$ & $46^{\circ} 01$ \\
\hline & randiba, $\mathrm{MG}$ & $\mathrm{Cla}$ & $20.1^{\circ} \mathrm{C}$ & & 1295 & 1097 & $17^{0} 51$ & $42^{\circ} 51$ \\
\hline
\end{tabular}

*Small populations (less than 45 lives trees); ${ }^{* *}$ Place that will be set up the new breeding population;

*** See Supplementary material. 
Supplementary Table 1. - Provenance of 7B082 - Anhembi Experimental Station.

\begin{tabular}{|c|c|c|c|c|c|}
\hline & Provenance & I otc & Iat $(S)$ & I cong $(E)$ & Alt (m) \\
\hline 01 & Nambucca SF/NSW & 9457 & $30^{\circ} 39^{\circ}$ & $153^{\circ} 00^{\prime}$ & 20 \\
\hline 02 & Wallingat/NSW & $\mathrm{B} 57-01$ & - & - & - \\
\hline 03 & Broken Bago SF/NSW & 6189 & $31^{\circ} 31^{\prime}$ & $152^{\circ} 40^{\prime}$ & 180 a 390 \\
\hline 04 & Dorrigo/NSW & $10845 / 138$ & $30^{\circ} 09^{\circ}$ & $152^{\circ} 42^{\prime}$ & 750 \\
\hline 05 & Woolgolga/NSW & 10718 & $29^{\circ} 48^{\circ}$ & $153^{\circ} 00^{\prime}$ & 120 \\
\hline 06 & Buladelah/NSW & 9453 & $32^{\circ} 25^{\prime}$ & $153^{\circ} 13^{\prime}$ & 75 \\
\hline 07 & Mt. Glorius/QLD & 6183 & $27^{\circ} 15^{\prime}$ & $152^{\circ} 40^{\circ}$ & 600 \\
\hline 08 & Fraser Island/QL & 9490 & $25^{\circ} 00^{\prime}$ & $153^{\circ} 00^{\prime}$ & 60 \\
\hline 09 & Gallagowan/QLD & $9490 / 2$ & $26^{\circ} 30^{\circ}$ & $152^{\circ} 20^{\prime}$ & 580 \\
\hline 10 & Bellthorpe/QLD & 10699 & $26^{\circ} 52^{\prime}$ & $152^{\circ} 42^{\prime}$ & 525 \\
\hline 11 & Mogi Guaçu & - & $28^{\circ} 18^{\prime}$ & $47^{\circ} 17^{\prime}$ & 600 \\
\hline 12 & Fraser Island/QLD & 12803 & $25^{\circ} 00^{\circ}$ & $153^{\circ} 00^{\circ}$ & 60 \\
\hline 13 & Limbabwe & - & - & - & - \\
\hline
\end{tabular}

Supplementary Table 2. - Provenances of the 7B103 - Anhembi Experimental Station.

\begin{tabular}{ccccccc}
\hline & Provenance & Lote & Lat (S) & Long (F) & Alt (m) & Seed trees \\
\hline 1 & Queens Lake SF & 13317 & $31^{\circ} 34^{\circ}$ & $152^{\circ} 47^{\circ}$ & 90 & 10 \\
2 & Black Mountain Road Kangaroo SF NW Moleton & 13324 & $30^{\circ} 08^{\circ}$ & $152^{\circ} 50^{\circ}$ & 550 & 10 \\
3 & Gallangowan SF QLD & 13451 & $26^{\circ} 27^{\circ}$ & $152^{\circ} 22^{\prime}$ & 610 & 7 \\
4 & Blackrock SF 10 Km Bcerburnm & 13537 & $26^{\circ} 57^{\circ}$ & $152^{\circ} 52^{\prime}$ & 30 & 5 \\
5 & Pt. 93 Zimbabwo & 6953 & - & - & - & - \\
6 & NW Cascade Dortigo District NSW & $10845 / 138$ & $30^{\circ} 09^{\circ}$ & $152^{\circ} 42^{\circ}$ & 750 & - \\
7 & $7,6 \mathrm{Km} \mathrm{S-SE} \mathrm{Ungoria} \mathrm{S} \mathrm{Yankee} \mathrm{Jack} \mathrm{Fraser} \mathrm{Island} \mathrm{QLD}$ & 12803 & $25^{\circ} 32^{\prime}$ & $153^{\circ} 00^{\circ}$ & 80 & 10 \\
8 & Gallagowan QLD & 9492 & $26^{\circ} 30^{\circ}$ & $152^{\circ} 20^{\circ}$ & 580 & 5
\end{tabular}

Supplementary Table 3. - Provenance of 167 - Itamarandiba population.

\begin{tabular}{cccccc}
\hline & Provenance & I.te & I at (S) & I.ong (F) & Alt (m) \\
\hline 1 & NW Gratlon, QLD & 6184 & - & - & - \\
2 & N. Grafton, NSW & 6186 & - & - & - \\
3 & S. Moruya, NSW & 6195 & $31^{\circ} 31^{\circ}$ & $152^{\circ} 40^{\circ}$ & $180-390$ \\
4 & Dorrigo D., NSW & 10845 & $30^{\circ} 09^{\circ}$ & $152^{\circ} 42^{\circ}$ & 750 \\
5 & & 9490 & & & \\
6 & Fraser Island, QLD & 6178 & $25^{\circ} 00^{\circ}$ & $153^{\circ} 00^{\circ}$ & 60 \\
7 & & 12227 & & & \\
8 & Gallagowan, QL.D & 9492 & $26^{\circ} 30^{\circ}$ & $152^{\circ} 20^{\circ}$ & 580 \\
9 & Bellthorpe, QLD & 10699 & $26^{\circ} 52^{\circ}$ & $152^{\circ} 42^{\circ}$ & 525 \\
\hline
\end{tabular}

in Australia was included for genotyping in this study as a reference population (Table 4 Supplementary materials). Trees were sourced from 5 geographically diverse provenances of $E$. pilularis. The 22 trees were classified into either a Northern $(n=6)$ or Southern $(n=16)$ region for some analyses (e.g. PCoA) based on an earlier study of population structure in $E$. pilularis (SHEPHERD et al., 2010).

\subsection{Microsatellite analysis}

The DNA was extracted from 100-150 mg of leaves of 150 individuals, using the DoYLE and Doyle (1987) method. The microsatellite loci were amplified as described by FARIA et al.
(2010). The analyzes were carried out using 21 microsatellites loci previously optimized for multiplex amplification systems for the genus Eucalyptus: EMBRA11, EMBRA63, EMBRA12, EMBRA204, EMBRA219, EMBRA333, EMBRA38, EMBRA210, EMBRA37, EMBRA1071, EMBRA179, ES-157, EMBRA1349, EMBRA175, EMBRA915, EMBRA2000, EMBRA1851, EMBRA1800, EMBRA6, EMBRA104 and EMBRA8. Microsatellite loci developed for $E$. grandis have been used successfully with $E$. pilularis in studies of genetic diversity in natural populations (SHEPHERD and RAYMOND, 2010; SHEPHERD et al., 2010). 


\subsection{Population genetic diversity}

Genetic diversity indexes, fixation index and coancestry coefficients were estimated for each population as well as a mean for all the Brazilian and all Australian populations. Allelic frequencies, mean number of alleles per locus $(A)$, allelic richness $(R)$, observed $\left(H_{o}\right)$ and expected heterozygosity $\left(H_{e}\right)$ and fixation index $(F)$ were estimated using the FSTAT program (GoudET, 2002). To test if the values were significantly different from zero, we used Monte Carlo permutation of alleles among individuals, and applied a Bonferroni correction for multiple tests. The mean individual fixation index $(F)$ and pairwise coancestry coefficient $\left(\theta_{i j}\right)$ between trees of the same provenance was estimated as in LOISELLE et al. (1995) using SPAGEDI 1.3 program (HARDY and VEKEMANS, 2002). The genetic differentiation among pairwise populations was calculated using the method of HEDRICK (2005) for microsatellite data:

$$
G_{S T}^{\prime}=\frac{G_{S T}\left(1+H_{s}\right)}{1-H_{S}}
$$

where $G_{S T}$ is the genetic differentiation among populations and $H_{S}$ is the mean genetic diversity within populations, estimated using the FSTAT program.

\subsection{Principal coordinates analysis and Mantel tests}

Principal coordinate analysis (PCoA) was performed in GenAlex (PEAKALL and SMouse, 2006) using the data for all loci and based on NEI's (1973) unbiased genetic distance matrix. The Mantel test (in GenALex) was used to test for inter-matrix correlations between the pairwise measure of coefficient coancestry (r), or relationship, and absolute pairwise differences in growth $(\mathrm{DBH})$, for individuals within a population. Pairwise differences in $r$ values were calculated in Excel to check relationship between growth within pairwise related trees.

Supplementary Table 4. - Natural origins for 24 Eucalyptus pilularis individuals of the reference population. Trees originated from five natural provenances. All provenances other than Conglomerate belong to the Southern Region. Two samples (10954 and 10960) from the Conglomerate provenance failed to re-amplify reliably therefore were left out of final genetic diversity estimates.

\begin{tabular}{|c|c|c|c|c|c|}
\hline Individual & Lote & Tree & Origin & Lat (\$) & Long $(\Gamma)$ \\
\hline 1 & 10728 & bbm007 & AUS NSW SF Moruya - Sth Batcmans Bay & $35^{\prime \prime} 47^{\prime}$ & $150^{\circ \prime} 04^{\circ}$ \\
\hline 2 & 10732 & bbbool & AUS NSW SF Bodalla - Narooma & $36^{\prime \prime} 08^{\prime}$ & $150^{\circ} 04^{\circ}$ \\
\hline 3 & 10735 & bbb004 & AUS NSW SF' Bodalla - Varooma & & \\
\hline 4 & 10736 & bbb005 & AUS NSW SF Bodalla - Narooma & & \\
\hline 5 & 10737 & bbb006 & AUS NSW SF Bodalla - Narooma & & \\
\hline 6 & 10738 & bbb007 & AUS NSW SF Bodalla - Narooma & & \\
\hline 7 & 10742 & en001 & AUS NSW SF Nullica W of Eden & $36^{\prime \prime} 59^{\prime}$ & $149^{\prime \prime} 54^{\prime}$ \\
\hline 8 & 10744 & $\operatorname{en}(003$ & AUS NSW SY' Nullica W of Eden & & \\
\hline 9 & 10746 & en005 & AUS NSW SF Nullica $W$ of Eden & & \\
\hline 10 & 10747 & 11006 & AUS NSW SF Nullica W of Eden & & \\
\hline 11 & 10750 & en009 & AUS NSW SF Nullica W of Eden & & \\
\hline 12 & 10931 & bby001 & AUS NSW SF Yerriyong Blackrange Rd Sth Nowra & $35^{\circ} 01^{\prime}$ & $150^{\circ} 34^{\circ}$ \\
\hline 13 & 10935 & bby005 & AUS NSW SF Yerriyong Blackrange Rd Sth Nowra & & \\
\hline 14 & 10936 & bby006 & AUS NSW SF Yerriyong Blackrange Rd Sth Nowra & & \\
\hline 15 & 10937 & bby007 & AUS NSW SF Yerriyong Blackrange Rd Sth Nowra & & \\
\hline 16 & 10938 & bby008 & AUS NSW SF Yerriyong Blackrange Rd Sth Nowra & & \\
\hline 17 & 10953 & conpyr 3 & AUS NSW SF Conglomerate & $35^{n} 07^{\prime}$ & $153^{\circ} 03^{\circ}$ \\
\hline 18 & 10954 & conpyrt & AUS NSW SF Conglomerate & & \\
\hline 19 & 10959 & conpyr9 & AUS NSW SF Conglomerate & & \\
\hline 20 & 10960 & conpyr 10 & AUS NSW SP Conglomerate & & \\
\hline 21 & 10961 & conpill & AUS NSW SF Conglomerate & & \\
\hline 22 & 10962 & conpil2 & AUS NSW St Conglomerate & & \\
\hline 23 & 10963 & conpil3 & AUS NSW SF Conglomerate & & \\
\hline 24 & 10966 & conpil6 & AUS NSW St' Conglomerate & & \\
\hline
\end{tabular}

See SHEPHERD et al. (2010) for more details on localities. 
Table 2. - Summary of measurement grow of Brazilian populations ( $\mathrm{H}$ is the total height; $\mathrm{DBH}$ is the diameter at breast height; Vol is the volume).

\begin{tabular}{|c|c|c|c|c|c|c|c|c|c|c|c|}
\hline & \multirow{3}{*}{ Provenance } & \multirow{3}{*}{$\begin{array}{l}\text { Planted } \\
\text { (yoar) }\end{array}$} & \multirow{3}{*}{$\begin{array}{l}\text { Logging } \\
\text { (times) }\end{array}$} & \multicolumn{4}{|c|}{ Provenance mean } & \multicolumn{3}{|c|}{ Biggest tree } & \multirow{3}{*}{$\begin{array}{l}\text { Age } \\
\text { (years) }\end{array}$} \\
\hline & & & & Density & 11 & DBII & Vol & 11 & DBHI & Vol & \\
\hline & & & & $\left(\right.$ tree/hat $\left.a^{-1}\right)$ & (n11) & $(\mathrm{m})$ & $\left(\mathrm{mo}^{3} \ln \mathrm{a}^{-1}\right)$ & (m) & $(\mathrm{m})$ & $\left(m^{3}\right)$ & \\
\hline 1 & Anh-82 & 1982 & 3 & 297 & 47 & 0.42 & 1008 & 55 & 0.64 & 8.9 & 31 \\
\hline 2 & Anh-103 & 1985 & 3 & 161 & 45 & 0.45 & 591 & 53 & 0.60 & 7.2 & 28 \\
\hline 3 & Anh-145 & 1989 & 2 & 350 & 34 & 0.34 & 709 & 49 & 0.50 & 4.5 & 24 \\
\hline 4 & Feena & 1919 & $\mathrm{NI}$ & 232 & 57 & 0.86 & 3500 & 60 & 1.51 & 48 & 94 \\
\hline 5 & Ita- 176 & 1984 & 3 & 219 & 29 & 0.30 & 330 & 34 & 0.42 & 2.4 & 29 \\
\hline 6 & Ita-498 & 1985 & 3 & 228 & 28 & 0.30 & 239 & 38 & 0.52 & 4.1 & 28 \\
\hline 7 & Ita-167 & 1984 & 3 & 281 & 24 & 0.28 & 281 & 27 & 0.40 & 1.8 & 29 \\
\hline 8 & Bir-K & 1976 & 0 & 500 & 51 & 0.48 & 2356 & 62 & 0.82 & 16.7 & 38 \\
\hline 9 & Bir-C & 1976 & 0 & 500 & 56 & 0.57 & 3300 & 63 & 0.88 & 18.2 & 38 \\
\hline
\end{tabular}

\section{Results}

\subsection{Genetic diversity and inbreeding levels in Brazilian populations were similar to the Australian reference populations}

Compared to the reference population of trees sampled directly in native stands in Australia, the levels of genetic diversity and inbreeding in the Brazilian populations were similar (Table 3 ). The total number of alleles $(k)$ ranged between 68 and 173, depending on the population; the highest number was detected in the
Ita-498 population. The number of private alleles for the Brazilian populations ranged from zero to 11. Ita-167, a multi-provenance trial, exhibited the highest number of private alleles. The two regional reference samples from natural stands in Australia were at the higher end of this range, 11 and 12 for the $\mathrm{N}$ and $\mathrm{S}$, respectively. The allelic richness $(R)$ ranged among population from 3.1 to 4.4 , with a mean of 3.9 . Observed heterozygosity $\left(H_{o}\right)$ ranged from 0.50 to 0.58 (mean of 0.57 ) and expected heterozygosity $\left(H_{e}\right)$ ranged from 0.60 to 0.75 (mean of 0.66 ).

Table 3. - Results of genetic diversity in the Eucalyptus pilularis populations.

\begin{tabular}{|c|c|c|c|c|c|c|c|}
\hline Samples & $n$ & $k$ & $P_{a}$ & $A \pm \mathrm{S} D$ & $R \pm \mathrm{SD}$ & $H_{o} \pm \mathrm{SD}$ & $H_{e}=\mathrm{SD}$ \\
\hline Anh-82 & 16 & 142 & 7 & $6.8 \perp 3.6$ & 4.011 .5 & $0.55 \perp 0.32$ & $0.69 \perp 0.24$ \\
\hline Anh-103 & 6 & 101 & 1 & $4.8 \perp 2.5$ & 3.911 .8 & $0.53 \perp 0.31$ & $0.66 \perp 0.33$ \\
\hline Anh-145 & 15 & 135 & 6 & $6.4 \pm 3.2$ & $3.8 \pm 1.5$ & $0.56 \pm 0.30$ & $0.64 \pm 0.28$ \\
\hline Fecna & 5 & 68 & 0 & $3.2 \pm 1.5$ & $3.1 \pm 1.3$ & $0.77 \pm 0.38$ & $0.60 \pm 0.26$ \\
\hline Ita-1 76 & 15 & 122 & 3 & $5.8 \pm 3.3$ & $3.6 \pm 1.6$ & $0.54 \pm 0.34$ & $0.61 \pm 0.32$ \\
\hline lta-498 & 22 & 173 & 9 & $8.2 \pm 4.7$ & $4.0 \pm 1.7$ & $0.54 \pm 0.31$ & $0.67 \pm 0.26$ \\
\hline Ita- 167 & 17 & 154 & 11 & $7.3+4.2$ & $3.9+1.6$ & $0.57+0.30$ & $0.64+0.28$ \\
\hline Bir-K & 15 & 138 & 8 & $6.6 \pm 3.5$ & $3.8 \pm 1.5$ & $0.56 \pm 0.30$ & $0.65 \pm 0.28$ \\
\hline Bir-C & 15 & 131 & 7 & $6.2 \pm 3.6$ & $3.7 \pm 1.5$ & $0.50 \pm 0.32$ & $0.64 \pm 0.27$ \\
\hline Brazil mean & 126 & 282 & - & $13.4=1.6$ & $8.8 \pm 4.0$ & $0.55 \pm 0.27$ & $0.70 \pm 0.05$ \\
\hline S-Aus & 16 & 150 & 12 & $7.1+3.5$ & $4.0+1.5$ & $0.54+0.25$ & $0.69+0.24$ \\
\hline $\mathrm{N}$-Aus & 8 & 125 & 11 & $6.0 \perp 2.8$ & $4.4 \pm 1.6$ & $0.58 \perp 0.31$ & $0.75 \perp 0.21$ \\
\hline Aust mean & 24 & 192 & - & $9.1 \pm 1.1$ & $8.7 \pm 4.0$ & $0.55 \pm 0.22$ & $0.72 \pm 0.05$ \\
\hline Overall mean & 150 & 131 & 6.8 & 6.2 & 3.9 & 0.57 & 0.66 \\
\hline
\end{tabular}


The population fixation index $(F)$ ranged from -0.11 to 0.81 (mean of 0.22 ), and was significantly larger than zero in all populations except Feena (Table 4). The mean of samples for the two Australian reference populations (0.29) was higher than the mean of all the Brazilian populations $(0.21)$. These relatively high $\mathrm{F}$ values for the Australian multi-provenance reference populations, was in contrast to earlier studies of samples from natural provenances that were analyzed on a single provenance basis (SHEPHERD et al., 2010).
2.2. Relationships among populations tended to reflect collections / experiments rather than natural origins

The most striking feature of the PCoA analysis was the tendency for introductions from the same institution to cluster together (Fig 1). In some cases the uniqueness (indicated by this distance in Eucalidan space to other populations) and clustering of populations held by the same organization, for example the two Biri populations, may have been explained by com-

Table 4. - Results of fixation index $(F)$ and coancestry coefficient $(\Theta)$ of Eucalyptus pilularis populations.

\begin{tabular}{lcccccccc}
\hline \multicolumn{1}{c}{ Samples } & $n$ & $F \pm 1.96 \mathrm{SE}$ & Mcdian & Min/Max & $n$ & $\Theta \pm 1.96 \mathrm{SE}$ & Mcdian & Min/Max \\
\hline Anh-82 & 16 & $0.24=0.08^{*}$ & 0.21 & $0.00 / 0.50$ & 120 & $0.04 \pm 0.02^{*}$ & 0.06 & $-0.14 / 0.40$ \\
Anh-103 & 6 & $0.26=0.15^{*}$ & 0.20 & $0.01 / 0.53$ & 15 & $0.08 \pm 0.04^{*}$ & 0.03 & $-0.05 / 0.29$ \\
Anh-145 & 15 & $0.15=0.06^{*}$ & 0.15 & $-0.03 / 0.41$ & 105 & $0.04 \pm 0.01^{*}$ & 0.04 & $-0.11 / 0.22$ \\
Feena & 5 & $-0.04 \pm 0.04$ & -0.06 & $-0.10 / 0.02$ & 10 & $0.26 \pm 0.04^{*}$ & 0.03 & $0.18 / 0.35$ \\
Ita-176 & 15 & $0.20-0.06^{*}$ & 0.20 & $-0.11 / 0.37$ & 105 & $0.10+0.02^{*}$ & 0.03 & $-0.11 / 0.38$ \\
Ila-498 & 22 & $0.22=0.06^{*}$ & 0.21 & $-0.05 / 0.50$ & 231 & $0.02 \pm 0.01^{*}$ & 0.14 & $-0.13 / 0.22$ \\
Ita-167 & 17 & $0.15=0.06^{*}$ & 0.16 & $-0.07 / 0.36$ & 136 & $0.04 \pm 0.01^{*}$ & 0.08 & $-0.12 / 0.39$ \\
Bir-K & 15 & $0.23=0.07^{*}$ & 0.23 & $0.03 / 0.44$ & 105 & $0.10 \pm 0.02^{*}$ & 0.24 & $-0.06 / 0.32$ \\
Bir-C & 15 & $0.35=0.06^{*}$ & 0.31 & $0.24 / 0.62$ & 105 & $0.15 \pm 0.02^{*}$ & 0.04 & $-0.05 / 0.41$ \\
Brazil mean & 126 & $0.21 \pm 0.03^{*}$ & 0.20 & $-0.11 / 0.62$ & 7875 & $0.07 \pm 0.01^{*}$ & 0.05 & $-0.14 / 0.41$ \\
\hline S-Aus & 16 & $0.26=0.08^{*}$ & 0.26 & $0.02 / 0.58$ & 120 & $0.05 \pm 0.01^{*}$ & 0.09 & $-0.13 / 0.35$ \\
N-Aus & 8 & $0.35=0.17^{*}$ & 0.21 & $0.13 / 0.81$ & 28 & $0.04 \pm 0.03^{*}$ & 0.01 & $-0.19 / 0.25$ \\
\hline Aust mean & 24 & $0.29=0.07^{*}$ & 0.26 & $0.02 / 0.81$ & 276 & $0.05 \pm 0.01^{*}$ & 0.03 & $-0.19 / 0.35$ \\
\hline Overall mean & 150 & $0.22=0.02^{*}$ & 0.21 & $-0.11 / 0.81$ & 11175 & $0.06 \pm 0.01^{*}$ & 0.05 & $-0.19 / 0.41$ \\
\hline
\end{tabular}

$n=$ sample size; $\mathrm{SE}=$ standard error. ${ }^{*} \mathrm{P}<0.05$.

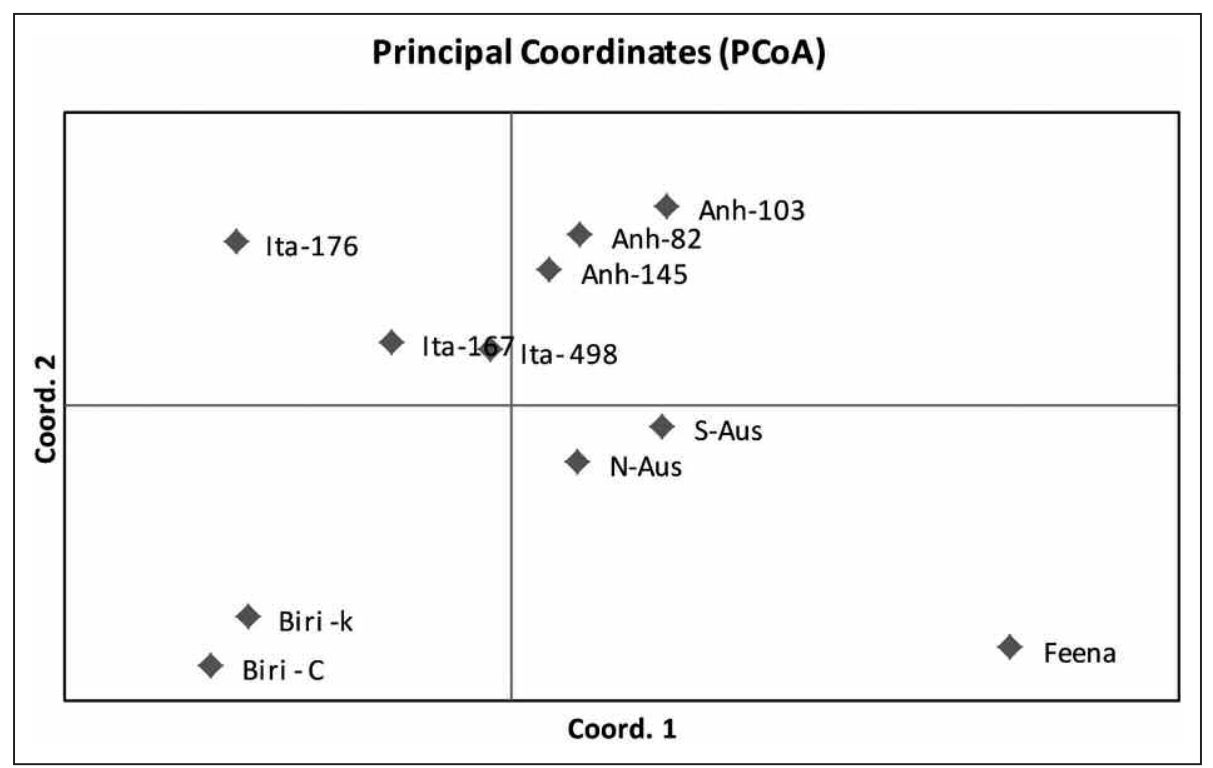

Figure 1. - Cluster analyses of population by PCoA via Covariance matrix. 
Table 5. - Pairwise $P_{\text {values }}$ (above diagonal) and genetic divergence ( $G_{S T}^{\prime}$, below diagonal) between 11 Eucalyptus pilularis populations.

\begin{tabular}{cccccccccccc}
\hline Sample & Anh-82 & Anh-103 & Anh-145 & Feena & Ita-176 & Ila-498 & Ita-167 & Bir-K & Bir-C & S-Aus & N-Aus \\
Anh-82 & - & 0.094 & 0.001 & 0.001 & 0.001 & 0.001 & 0.001 & 0.001 & 0.001 & 0.001 & 0.010 \\
Anh-103 & 0.031 & - & 0.001 & 0.005 & 0.001 & 0.001 & 0.001 & 0.001 & 0.001 & 0.001 & 0.037 \\
Anh-145 & 0.079 & 0.080 & - & 0.001 & 0.001 & 0.005 & 0.001 & 0.001 & 0.001 & 0.001 & 0.006 \\
Feena & 0.298 & 0.261 & 0.295 & - & 0.001 & 0.001 & 0.001 & 0.001 & 0.001 & 0.001 & 0.043 \\
Ita-176 & 0.212 & 0.219 & 0.196 & 0.500 & - & 0.001 & 0.001 & 0.001 & 0.001 & 0.001 & 0.007 \\
Ita-498 & 0.079 & 0.120 & 0.048 & 0.290 & 0.184 & - & 0.001 & 0.001 & 0.001 & 0.001 & 0.003 \\
Ita-167 & 0.139 & 0.198 & 0.082 & 0.353 & 0.127 & 0.048 & - & 0.001 & 0.001 & 0.001 & 0.004 \\
Bir-K & 0.266 & 0.344 & 0.269 & 0.446 & 0.226 & 0.202 & 0.195 & - & 0.001 & 0.001 & 0.010 \\
Bir-C & 0.355 & 0.423 & 0.331 & 0.548 & 0.265 & 0.279 & 0.274 & 0.139 & - & 0.001 & 0.010 \\
S-Aus & 0.075 & 0.149 & 0.128 & 0.236 & 0.269 & 0.105 & 0.179 & 0.216 & 0.286 & - & 0.006 \\
N-Aus & 0.183 & 0.221 & 0.205 & 0.362 & 0.227 & 0.201 & 0.218 & 0.244 & 0.300 & 0.141 & - \\
\hline
\end{tabular}

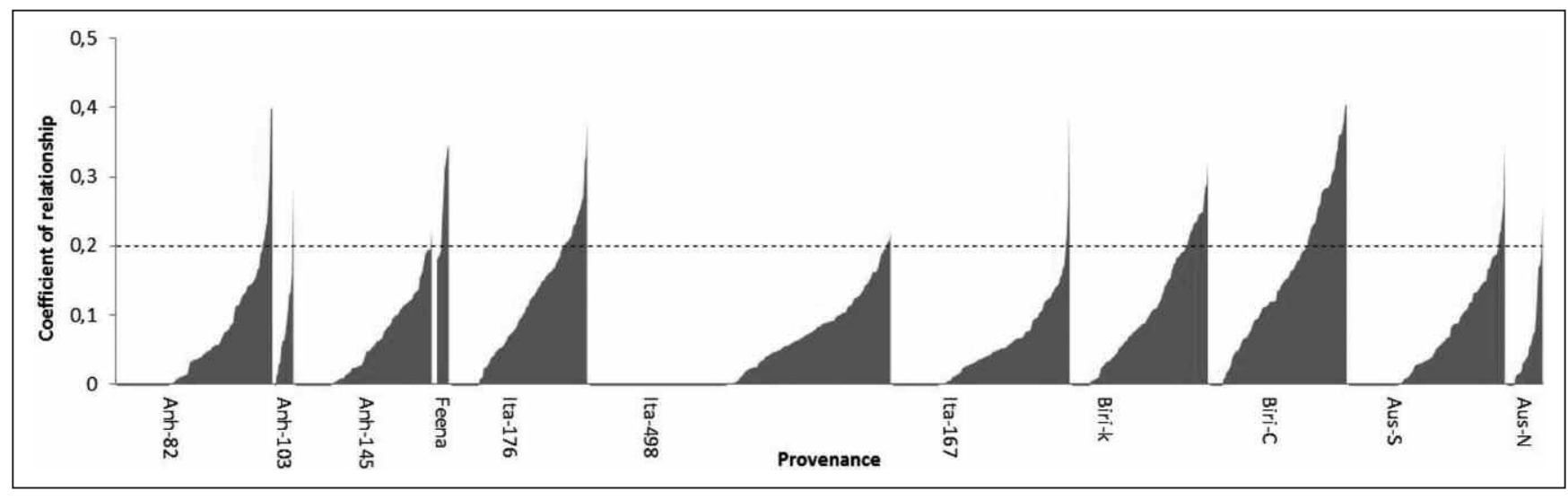

Figure 2. - Pairwise coefficient of relationship within 11 populations of blackbutt.

monality in the sources of the materials. For example, these two Biri populations are singleprovenance trials (based on Coopernook or Kiwarrak provenances), which is an example where the natural origins of the trees are known to have come from neighbouring provenances. Two multi-provenance Anhe populations, share some common provenance origins (and the same seedlots), thus again they might be expected to be related (Tables 1 and 2 of the supplementary material). All three Anhe populations contained trees from the Frazer Is provenance (The anhe 145 population was all derived from the Frazer island provenance) thus again commonality in geographic origins may be causing clustering in these cases.

In other cases, for example when the individuals from the Australian reference population were classified into a northern and southern region, the two populations still tended to cluster relatively close together. This was unex- pected given that this regional difference was the strongest degree of genetic structuring evident in E. pilularis (SHEPHERD et al., 2010). In addition other expected relationships among populations sharing commonality in geographic origins were also not evident. For example the Northern Australian population was expected to show affinity with Bir-Coop and Bir-K populations, which are geographically more proximal than the Southern Australian population. Thus in general it was thought that the relationship evident by PCoA did not reflect relationships in the provenance origins that might be expected where populations exhibit isolation by distance (IBD). To some degree, the lack of evidence for IBD in the study may be due to the overall low levels of genetic structure evident in this species (SHEPHERD et al., 2010a; 2010b), but may also reflect difficulty in estimating measures of genetic differentiation based on small sample sizes, or that due to the shared parentage among trees in the introduced populations 
(due to common seedlots) which may be confounding and masking subtle differences among geographic sources. The PCoA, however, was also useful in identifing populations that tended to be more distinct, such as the Ita-176 population, which was quite distinct from the other

Supplementary Table 5. - Coancestry coefficient (below diagonal) and diameter at breast height (DBH) growth difference in $\mathrm{cm}$ (above diagonal) between trees from Anhembi 82 (A) and Itamarandiba 498 (B) populations.

A - Anhembi

\begin{tabular}{ccccccccccccccccc}
\hline Trees & 1 & 2 & 3 & 4 & 5 & 6 & 7 & 8 & 9 & 10 & 11 & 12 & 13 & 14 & 15 & 16 \\
\hline 1 & - & -12.57 & -9.23 & -11.46 & -7.32 & -10.50 & -10.50 & -10.82 & -13.85 & -14.96 & -14.64 & -17.51 & -7.96 & -17.83 & -20.37 & -16.39 \\
2 & 0.19 & - & 3.34 & 1.11 & 5.25 & 2.07 & 2.07 & 1.75 & -1.27 & -2.39 & -2.07 & -4.93 & 4.62 & -5.25 & -7.80 & -3.82 \\
3 & 0.40 & 0.16 & - & -2.23 & 1.91 & -1.27 & -1.27 & -1.59 & -4.62 & -5.73 & -5.41 & -8.28 & 1.27 & -8.59 & -11.14 & -7.16 \\
4 & 0.07 & 0.12 & 0.07 & - & 4.14 & 0.95 & 0.95 & 0.64 & -2.39 & -3.50 & -3.18 & -6.05 & 3.50 & -6.37 & -8.91 & -4.93 \\
5 & 0.03 & -0.05 & 0.09 & 0.01 & - & -3.18 & -3.18 & -3.50 & -6.53 & -7.64 & -7.32 & -10.19 & -0.64 & -10.50 & -13.05 & -9.07 \\
6 & 0.04 & -0.02 & 0.06 & 0.07 & 0.01 & - & 0.00 & -0.32 & -3.34 & -4.46 & -4.14 & -7.00 & 2.55 & -7.32 & -9.87 & -5.89 \\
7 & 0.21 & 0.01 & 0.13 & 0.04 & -0.01 & 0.04 & - & -0.32 & -3.34 & -4.46 & -4.14 & -7.00 & 2.55 & -7.32 & -9.87 & -5.89 \\
8 & 0.13 & 0.01 & 0.17 & -0.09 & -0.03 & 0.08 & 0.23 & - & -3.02 & -4.14 & -3.82 & -6.68 & 2.86 & -7.00 & -9.55 & -5.57 \\
9 & 0.06 & -0.01 & 0.06 & 0.15 & 0.03 & 0.00 & -0.03 & 0.01 & - & -4.14 & -3.82 & -3.66 & 5.89 & -3.98 & -6.53 & -2.55 \\
10 & -0.12 & -0.06 & -0.03 & 0.40 & -0.02 & 0.08 & 0.10 & 0.05 & 0.17 & - & 0.32 & -2.55 & 7.00 & -2.86 & -5.41 & -1.43 \\
11 & 0.08 & 0.06 & 0.01 & 0.22 & -0.03 & 0.12 & 0.11 & 0.03 & 0.14 & 0.20 & - & -2.86 & 6.68 & -3.18 & -5.73 & -1.75 \\
12 & 0.04 & 0.14 & 0.00 & 0.14 & -0.05 & 0.04 & -0.05 & -0.03 & -0.01 & 0.05 & 0.05 & - & 9.55 & -0.32 & -2.86 & 1.11 \\
13 & -0.09 & -0.05 & -0.06 & 0.09 & -0.07 & 0.04 & 0.04 & 0.11 & 0.02 & 0.30 & 0.15 & -0.04 & - & -9.87 & -12.41 & -8.44 \\
14 & -0.10 & -0.08 & -0.11 & 0.13 & 0.01 & 0.15 & -0.06 & -0.06 & 0.04 & 0.15 & 0.06 & 0.01 & 0.04 & - & -2.55 & 1.43 \\
15 & 0.19 & -0.03 & 0.26 & -0.06 & 0.08 & -0.01 & 0.13 & 0.11 & -0.01 & -0.07 & 0.01 & -0.09 & -0.08 & 0.00 & - & 3.98 \\
16 & 0.05 & 0.04 & 0.00 & -0.07 & -0.04 & -0.14 & 0.05 & -0.05 & 0.08 & -0.01 & 0.00 & -0.01 & -0.09 & 0.01 & 0.05 & - \\
\hline
\end{tabular}

Note: Anh-82 is a main multiprovenance Eucalyptus pilularis population in the Anhembi Experimental Station, where will be set up the new generation breeding population of the specie with the plustrees harvested in this study.

\section{B -Itamarandiba}

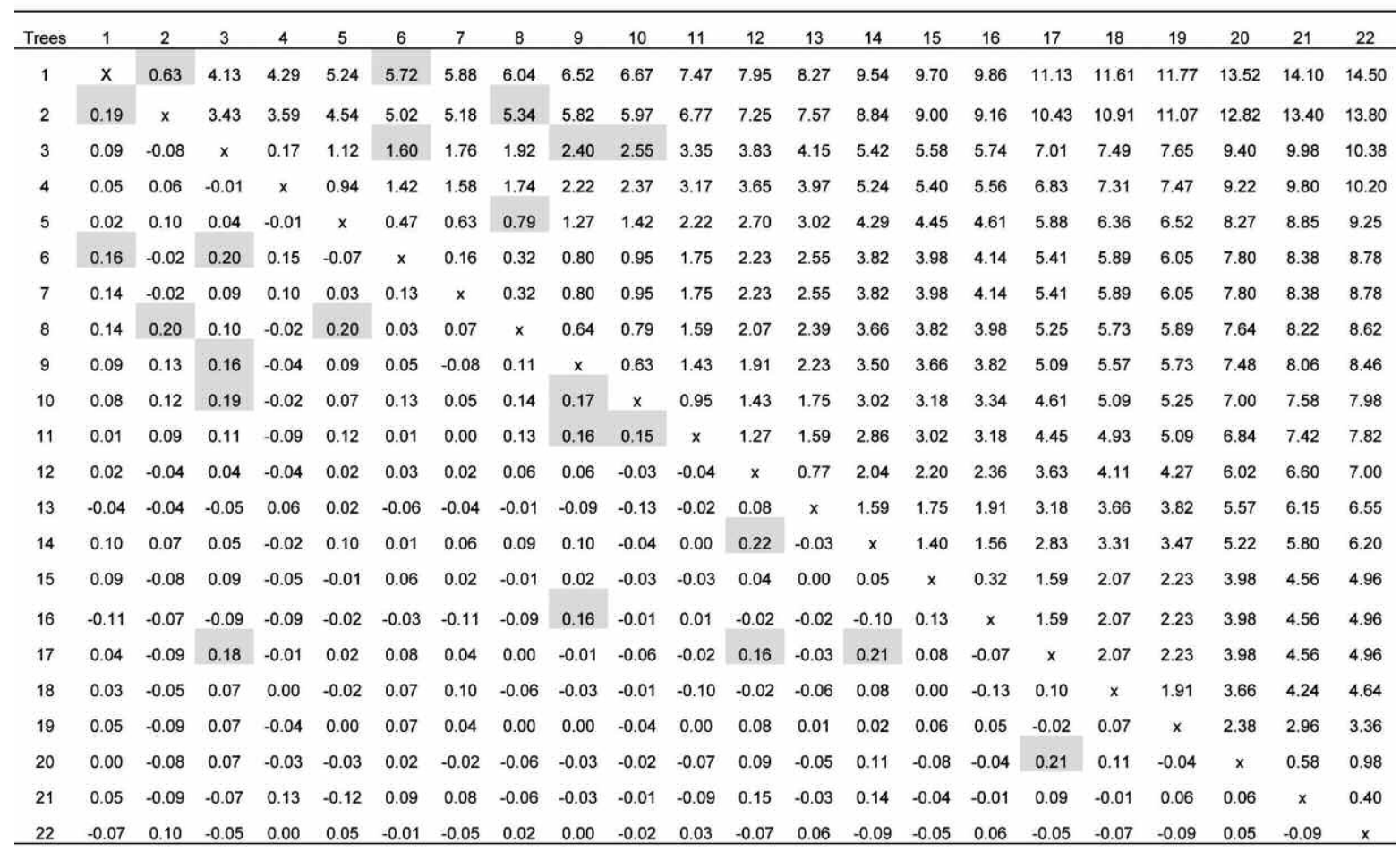

Note: Ita-498 was the multiprovenance Eucalyptus pilularis population that showed lower number of related plus trees in our study. 
two populations from Itamarandiba. The Feena population was an outlier and quite distinct from all other populations. Such information may be useful for stratifying sampling when trying to manage inbreeding.

Pairwise $G_{S T}^{\prime}$ values were also used to quantify the degree of genetic differentiation among populations. The pairwise genetic differentiation $\left(G_{S T}^{\prime}\right)$ among populations ranged from 0.031 to 0.548 and were significantly different to zero in all but one case (two Anh populations Table 5).

\subsection{Better performing trees in an experiment did not tend to be related}

The lack of experimental design information or tree identities precluded comparison of performance of provenances, nonetheless we note that within an experiment, the selected plus trees were $20 \%$ and $54 \%$ higher in $\mathrm{H}$ and $\mathrm{DBH}$ than the population means. Again, the Feena population tended to be different as its biggest tree was only $5 \%$ taller than the average of selected plus trees, but its $\mathrm{DBH}$ was $75 \%$ higher $(1.5 \mathrm{~m}$ versus $0.86 \mathrm{~m}$ of the average of trees inside Feena).

Several plus-tree are related in two Brazilian populations, and in general, plus-trees tended to show degrees of relationship to other trees in the same population (Fig. 2). Ita-498 experiment was an exception as the plus trees selected here did not tend to be more related than the population average.

The pairwise coancestry coefficient $(\Theta)$ was significant higher than zero in all populations and ranged from -0.19 to 0.41 (mean $=0.06)$. In the Feena population, all $\Theta$ values were higher than zero (mean of 0.26 , ranging from 0.18 to $0.35)$.

The degree of correlation between the pairwise ancestry coefficient and the difference in growth performance for pairs of trees was tested in a Mantel test on a population basis. The test was not significantly different from zero for the populations Anh-82 and Ita-498, two multi-provenance populations, with contrasting results regarding the degree of related between selected plus trees (Table 5 Supplementary material). This suggested that related plus trees did not tend to show similar growth performance in the field.

\section{Discussion}

\subsection{Genetic diversity and interpretation of $F$ from admixtures}

Genetic diversity of introduced Brazilian populations was similar to the broad-based reference population from native stands in Australia. This was positive news from the point of view of providing a broad genetic base for reinvigorating breeding of $E$. pilularis in Brazil.

The fixation index $(F)$, however, was significant higher than zero in all except one population analyzed in this study. This included the two Australian multi-provenance reference populations which when analyzed previously as single provenances, did not exhibit high fixation indices (SHEPHERD et al., 2010). This suggests the high $F$ values detected in this study are a consequence of studying population admixtures i.e. due to a Wahlund effect where substructure causes lower frequencies of heterozgyotes than expected for a random mating population (HARTL, D.L., 2000). This effect may be evident where populations that have been isolated come back in contact, or where populations are combined artificially. Most of the Brazilian populations studied here are multi-provenance collections thus combine across populations that are unlikely to be panmixtic. The lower $F$ value for the Freena population probably reflects its single-provenance origins. The Feena population is believed to be a second generation population (seeds from Campania Paulista in accordance with the records), but its low value suggests little inbreeding has occurred among the selections from the first generation. The relatively high $F$ values for the reference populations in this study, also multi-provenance populations, but which did not exhibit large $F$ when analyzed as single-provenance populations, was also consistent with a Wahlund effect.

Given the lack of evidence of inbreeding in natural populations of E. pilularis (SHEPHERD et al., 2010), it seems likely that the relatively high fixation indices evident in the current study are a consequence of studying derived admixtures. Although inbreeding cannot be ruled out at this stage, generally where seed collections were made according to appropriate rules and there has not been regular mating schemes applied, as for this first generation of trees introduced into Brazil, inbreeding should not be large. Overall, from this we conclude 
there is little evidence for inbreeding in current populations introduced into Brazil.

\subsection{Genetic relationships among Brazilian populations}

As expected, genetic relationships among introduced Brazilian populations did not accord with the geographic origins. Instead relationships tended to cluster by source organization. This suggests the subtle geographic based structure due to isolation by distance evident in natural populations has been masked in this study by the similarly in populations as a consequence of the overlap in seedlots introduced into Brazil by the same organization, and the admixture caused by the introduction of multiple provenances in the introductions by the same organization. These findings suggest it will be important to stratify sampling for the new breeding population across collections from the different institutions to maximize genetic breadth in the next generation. This analysis of the relationship amongst introductions also identified outlier populations such as Feena and Biri, which can help with sampling designs.

The lack of structuring along geographic origins suggested that it will be unlikely that the provenance origins for individual trees can be recovered where they are unknown. Given the subtle genetic structuring evident broadly across E. pilularis in microsatellite markers (SHEPHERD et al., 2010), this was always going to be challenging. Much larger numbers of individuals in reference populations and many more genetic loci would appear to be required to have any hope of reconstructing provenance origins.

Mantel tests were used to test for inter-correlation amongst a matrix of within-population pairwise $r$ values and pairwise differences in DBH. Such a correlation may be evident assuming growth is largely additively genetically controlled, and therefore a function of heritability and relatedness (KUMAR and RICHARDSON, 2005), however no relationship was evident in our study for our multi-provenance populations. Therefore, despite the lack of pedigree information for many of the selected plus trees in the current study to help manage inbreeding, the results suggest potential inbreeding effects arising from mating between relatives have been largely avoided (HARDNER and POTTS, 1995; KUMAR and RICHARDSON, 2005).
All sampled populations had related individuals, but in the Brazilian populations, the relatedness measured by mean pairwise coancestry coefficient $(\Theta)$ was in general higher than in Australian populations. The cause of higher coancestry may be the family structure in the Brazilian material, thus in these cases the minimum expected coancestry coefficient is 0.125 (half-sibs). The highest coancestry values were detected in Feena population, where all $\Theta$ values were all higher than zero (mean of 0.26 ), suggesting that these six trees originated from the same seed tree and may be related as fullsibs $(\Theta=0.25)$. The presence of related individuals among progeny from a selected plus tree in the breeding programs have strong implications for next generation of selections. As already mentioned, eucalypt species exhibit inbreeding depression, and mating among relatives may result in individuals with low performance for economical important traits such as growth, survival, adaptation and resistance to disease (HARDNER and PoTTS, 1995). Thus, it is important to increase the number of selected plus trees or effective population size of breeding populations to decrease the relatedness within these populations and, consequently decrease the probability of inbreeding occurring in next generations of selection.

\subsection{Selecting adapted sources of E. pilularis for planting in Brazil}

In general $E$. pilularis grew well across a range of sites in Brazil. It was clear that given sufficient time (i.e. some trees were 94 years of age), trees could be of similar stature to those found in native forests (BROOKER and KLEINIG, 2006). Large trees were obtained from a range of provenances, including provenances such as Frazer Island (DBH of $0.5 \mathrm{~m}$ at 24 years of age) which was regarded as a poor performing provenance on more productive trial sites in Australia (Burgess, 1975; CARNEGIE et al., 2004). These trees were found in the Anhembi experimental station trial, where the initial result of old multi-provenance populations (Anh-82 and Anh-103) drove the genetic improvement program to install a new population (Anh-145) with only progenies from Frazer Island (personal comments of Prof. Mario Ferreira). Anhembi Experimental Station has a sand soil and the populations were planted without intensive silviculture (i.e. no fertilization, weed control, etc), as is current practice in Brazil 
(GonÇAlves et al., 2013; Silva et al., 2013). Populations in Biritiba Mirim showed good growth, (provenances Coopernook and Kiwarrak), which is in accordance with the general observation that trees adapted to more productive natural environments, are able to take advantage of good conditions at a planting site (BURGESS, 1975; CARNEGIE et al., 2004). However due to the differences among trials (age, climatic conditions, etc) in our study, it was not possible to compare Coopernook with other provenances like Frazer Island where poor growth was expected.

\section{Acknowledgement}

The authors would like to thank the FAPESP, the companies Aperam and Suzano for the support and reviewers for their comments and help in our study.

The authors Alexandre Magno SEbBenn and DARIO GRATtaPAGLIA would like to thank the Conselho Nacional de Desenvolvimento Cient1fico e Tecnologico (CNPq) for being granted a Research Fellowship.

\section{References}

Alcorn, P. J., J. Bauhus, R. G. B. Smith, D. Thomas, R. I. JAMES and A. NicotrA (2008): Growth response following green crown pruning in plantation-grown Eucalyptus pilularis and Eucalyptus cloeziana. Can. J. For. Res. 38, 770-781.

Brooker, M. I. H. and D. A. KLEINIG (2006): Field Guide to Eucalypts. Vol.1. South-eastern Australia. $3^{\text {rd }}$ ed. Bloomings, Melbourne, Australia.

Burgess, I. P. (1975): A provenance trial with blackbutt: 9 years results. Aust For. Res., 7, 1-9.

CARnegie, A. J., I. G. Johnson and M. Henson (2004): Variation among provenances and families of blackbutt (Eucalyptus pilularis) in early growth and susceptibility to damage from leaf spot fungi. Can J. For. Res., 34, 2314-2326.

Doyle, J. J. and J. L. DoYLE (1987): A rapid DNA isolation procedure for small quantities of fresh leaf tissue. Phytochem Bull, 9, 11-15

El-Kassaby, Y. A., E. P. CAPPa, C. LiewlaksaneeYANAWIN, J. KLÁPS̆TE and M. LSTIBU゚REK (2011): Breeding without breeding: is a complete pedigree necessary for efficient breeding? PLoS One, 6, e25737.

Faria, D. A., E. M. C. Mamani, M. R. Pappas, G. J. PAPPAS and D. Grattapaglia (2010): A selected set of EST-derived microsatellites, polymorphic and transferable across 6 Species of Eucalyptus. J. Hered., 101, 512-520.
Gonçalves, J. L. M., C. A. Alvares, A. R. Higa, L. D. Silva, A. C. Alfenas, J. Stahl, S. F. B. FerRaz, W. P. Lima, P. H. S. Brancalion, A. Hubner, J. P. D. Bouillet, J. P. Laclau, Y. Nouvellon and D. EPRON (2013): Integrating genetic and silvicultural strategies to minimize abiotic and biotic constraints in Brazilian eucalypt plantations. Forest. Ecol.. Manage., 301, 6-27.

GoudET, J. (1995): Fstat (Version 2.9.3.2.): A computer program to calculate F-statistics. J Hered 86: $485-486$.

Hardner, C. M. and B. M. PotTs (1995): Inbreeding depression and changes in variation after selfing in Eucalyptus globulus ssp. globulus. Silvae Genet 44: 46-54.

HARDY, O. and X. VEKEMANS (2002): SPAGeDI: a versatile computer program to analyze spatial genetic structure at the individual or population levels. Mol. Ecol. Notes, 2: 618-620.

HartL, D. L. (2000): A Primer of Population Genetics. $3^{\text {nd }}$ ed. Sinauer Associates Inc, Sunderland, Massachsetts.

HARWOOD, C. (2011): New introductions - doing it right. In: Developing a eucalypt resource. Learning from Australia and elsewhere. Ed. J. WALKER. Wood Technology Research Centre, University of Canterbury, Christchurch, New Zealand, 125-136.

Jaleel, C. A., P. Manivannan, A. Wahid, M. Farooq, R. Somasundaram and R. PanneErselvam (2009): Drought stress in plants: a review on morphological characteristics and pigments composition. Int. J. Agric. Biol., 11, 100-105.

KumAR, S. and T. E. RichaRdson (2005): Inferring relatedness and heritability using molecular markers in radiata pine. Mol. Breed., 15, 55-64.

NEI, M. (1973): Analysis of gene diversity in subdivided populations. P.N.A.S., 70, 3321-3323.

PAszTor, Y. P. C. (1974): Teste de procedências de Eucalyptus pilularis SM na região de Mogi-Guaçu. IPEF, 8, 69-93.

PeAKall, R. and P. E. SMouse (2006): GENALEX 6: genetic analysis in Excel. Population genetic software for teaching and research. Mol Ecol Notes. 6, 288-295.

Silva, P. H. M., F. Poggiani, P. L. Libardi and A. N. GonÇALVEs (2013): Fertilizer management of eucalypt plantations on sandy soil in Brazil: Initial growth and nutrient cycling. For. Eco. Manage., 301, 67-78.

Shepherd, M., T. R. Sexton, D. Thomas, M. Henson and R. J. HENRYA (2010): Geographical and historical determinants of microsatellite variation in Eucalyptus pilularis. Can. J. For. Res., 40, 1051-1063.

ShepherD, M. and C. RAYMond (2010): Species differentiation and gene flow in the Blackbutts (genus Eucalyptus subgenus Eucalyptus section Pseudophloius). Conserv. Genet., 11, 1965-1978.

Stape, J. L., D. Binkley and M. G. RYAN (2008): Production and carbon allocation in a clonal Eucalyptus plantation with water and nutrient manipulations. For. Ecol. Manage., 255, 920-930. 